\title{
A PROBLEM ON ENDOMORPHISMS OF PRIMARY ABELIAN GROUPS
}

\author{
ROBERT W. STRINGALL
}

In this note, an example is constructed which gives a negative answer to the following question posed by R. S. Pierce [2, p. 367].

Let $B$ be a basic $p$-group, and $\bar{B}$ the torsion completion of $B$. Let $P$ be a subgroup of the socle of $\bar{B}$ such that $B[p] \subseteq P$. Let $\alpha$ be an endomorphism of $\bar{B}$ such that $\alpha(P) \subseteq P$. Does there exist a pure subgroup $G$ of $\bar{B}$ such that $B \subseteq G, G[p]=P$ and $\alpha(G) \subseteq G$ ?

Let $N$ be the set of positive integers and let $B=\sum_{i \in N} \oplus B_{i}$ be a standard basic group with projections $\rho_{i}: B \rightarrow B_{i}$. Note that $B_{i}=\left\{b_{i}\right\}$ is cyclic and of order $p^{i}$. For notational convenience, let $c_{i}=p^{i-1} b_{i}$. Let $y=\sum_{i \in N} c_{3 i}$ and let $P$ be the subgroup of $\bar{B}[p]$ generated by $B[p]$ and $\{y\}$. Let $\alpha$ be the endomorphism of $\bar{B}$ determined by the conditions:

$$
\alpha\left(b_{i}\right)=\left\{\begin{array}{cl}
b_{i}+p^{2} b_{i+1} & \text { if } i=3 n \\
p b_{i+1} & \text { if } i=3 n+1 \\
b_{i} & \text { if } i=3 n+2
\end{array}\right.
$$

It follows that $\alpha(y)=y$ and that $\alpha(B) \subseteq B$. Thus,

$$
\alpha(P)=\alpha(B[p]+\{y\}) \subseteq \alpha(B[p])+\alpha(\{y\}) \subseteq B[p]+\{y\}=P .
$$

Suppose there is a pure subgroup $G$ of $\bar{B}$ such that $B \subseteq G, G[p]=P$ and $\alpha(G) \subseteq G$. Let $x=\sum_{i \in N ; i>2} a_{i} b_{i} \in G$ be such that $p x=y$. There must be such an element $x$ since $B \subseteq G, G$ is pure in $\bar{B}$ and since the height of $y$ in $\bar{B}$ is 2 . Now for each $i \in N, \rho_{3 i}(\alpha(x)-x)=0$ since $\rho_{3 i}(x)=a_{3 i} b_{3 i}=\rho_{3 i} \alpha\left(a_{3 i} b_{3 i}\right)=\rho_{3 i} \alpha(x)$. Also, $\alpha(x)-x \in P$ since $p(\alpha(x)-x)$ $=\alpha(y)-y=0$. Consequently, by the definition of $P, \alpha(x)-x \in B$. It follows that $\rho_{i}(\alpha(x)-x)=0$ for all but a finite number of indices $i \in N$. Now, since

$$
\begin{aligned}
\alpha(x) & =\sum_{i \in N} a_{3 i} \alpha\left(b_{3 i}\right)+\sum_{i \in N} a_{3 i+1} \alpha\left(b_{3 i+1}\right)+\sum_{i \in N} a_{3 i+2} \alpha\left(b_{3 i+2}\right) \\
& =\sum_{i \in N}\left(a_{3 i} b_{3 i}+a_{3 i} p^{2} b_{3 i+1}+a_{3 i+1} p b_{3 i+2}+a_{3 i+2} b_{3 i+2}\right), \\
\alpha(x)-x & =\sum_{i \in N}\left(a_{3 i} p^{2}-a_{3 i+1}\right) b_{3 i+1}+\sum_{i \in N} a_{3 i+1} p b_{3 i+2} .
\end{aligned}
$$

Received by the editors November 4, 1965. 
Therefore

$$
\rho_{3 i+2}(\alpha(x)-x)=a_{3 i+1} p b_{3 i+2}
$$

and

$$
\rho_{3 i+1}(\alpha(x)-x)=\left(a_{3 i} p^{2}-a_{3 i+1}\right) b_{3 i+1} .
$$

Thus, if $\rho_{i}(\alpha(x)-x)=0$ for almost all $i \in N$, then $p^{3 i+1}$ divides $a_{3 i+1}$ and, consequently, $p^{3 i+1}$ divides $a_{3 i} p^{2}$ for almost all indices $i \in N$. This implies that $p^{3 i-1}$ divides $a_{3 i}$ for almost all $i$. Thus,

$$
\rho_{3 i}(y)=p \rho_{3 i}(x)=p a_{3 i} b_{3 i}=0
$$

for almost all $i \in N$, contradicting the definition of $y$. Therefore, no such pure subgroup $G$ exists.

\section{REFERENCES}

1. L. Fuchs, Abelian groups, Publ. House Hungar. Acad. Sci. Budapest, 1958.

2. R. S. Pierce, Homomorphisms of primary abelian groups, Topics in Abelian Groups, Chicago, Ill., 1963.

University of California 